\title{
EFFECT OF NITROGEN FERTILIZER AND SUPPLEMENTARY IRRIGATION ON YIELD AND QUALITY OF SOME LOCAL BREAD WHEAT VARIETIES
}

\author{
Mohammed A. Al-Noori Ahmed S. Khalaf \\ College of Agric. and Foresty, Mosul Univ., Iraq
}

\begin{abstract}
Wheat production under climate of northern Iraq depends mainly on annual rain quantity and its distribution. Generally the annual rate is low and poorly distributed ,as a result the yield is low and unstable. Accordingly it has been suggested that small amounts of supplementary irrigation (SI) can alleviate the adverse effects of such unfavorable rain pattern, moreover the optimum dose of nitrogen fertilization is an important factor boosting wheat production and improving quality. The purpose of this study was to investigate the effect of nitrogen fertilizer and abridged (SI) on yield and grain quality. Field experiments were performed during the growing season 2003-2004 at two locations laying out as split-split plot design in RCBD, involving four promising bread cultivars as sub-sub plot, two levels of $\mathrm{N}$ fertilizer (zero and $80 \mathrm{~kg} \mathrm{~N} / \mathrm{ha}$ ) as sub-plot under rainfall alone and with (SI) as a main plot added at booting and heading stages at a rate of $25 \mathrm{~mm}$ for each stage. The results showed that $\mathrm{N}$ fertilizer had a significant effect on grain yield, grain protein content at both locations. The (SI) affected significantly number of grain/spike, grain yield, the interactions between factors were significant for most characters.
\end{abstract}

\section{INRODUCTION}

Wheat (Triticum aestivum L.) is the major cereal crop grown in Iraq, especially at northern regions mostly under rain fed condition therefore wheat production depends greatly on annual rainfall amount and distribution. At Ninevah governorate where the study was conducted the annual rainfall is about 350-500 mm and were usually poorly distributed, so the yield are rather low and unstable , the average of wheat yield under rainfall conditions for 1993-2003 was $179 \mathrm{~kg} /$ donum with great scattering range comprise from 51.3 to $415.3 \mathrm{~kg} / \mathrm{don}$. in 1999 and 2001, respectively (Statistic Issue, 2003), information of previous studies showed that small amount of supplemental irrigation may alleviate the adverse effects of unfavorable rain pattern and leads to improve and stabilize wheat yield (Garabet et al., 1998b; Oweis et al., 1998; Oweis et al.,2000; Adary et al., 2002). The aim of adding (SI) was to raise the productivity of wheat which suffering from shortage of moisture, for example the rain water use efficiency was raised from 9.1 to $15 \mathrm{~kg} / \mathrm{ha} / \mathrm{mm}$ by adding $68 \mathrm{~mm}$ of (SI) in Iraq (Adary et al., 2002) other results showed that addition of $73 \mathrm{~mm}$ of (SI) raised the productivity of wheat to about $67 \%$ in Jordan, adding $36 \mathrm{~mm}$ of (SI) raised the productivity to about $46 \%$ in Syria (Nosaif, 2002), (SI) as a practice is still unusual by local farmers, and they are unfamiliar with such system, therefore researches needs to optimize such practices. In addition to (SI), nitrogen fertilization was extensively used to improve yield and grain quality of wheat, but the response for nitrogen depends on rainfall amount or on irrigation, usually 
sufficient amount of rain or irrigation raise the nitrogen use efficiency (Engel $e t$ al.,1993; Engel et al., 2001; Garabet et al. 1998a). Part of $\mathrm{Ph}$. D thesis of the first author Received 29/5/2006, accepted 16/8/2006

Furthermore, the response of wheat to nitrogen and irrigation depends on cultivars (Ottman et al., 2000). The agriculture extension should play a role in transferring such results to farmers for adoption. According to literatures reported studies of the relationships between (SI) and nitrogen fertilization on wheat grain quality are rare, therefore the objective of this investigation was to study the effect of nitrogen fertilization and accompanying irrigation and their interaction on some grain quality properties and growth in addition to yield of four local promising bread wheat varieties (three of them are recently released).

\section{MATERIALS AND METHODS}

Field trails were carried out in 2003-2004 growing season at two locations (Mosul and Telkaef $30 \mathrm{~km}$ north Mosul), The experiments were designed as a split-split plot in RCBD with three replications, each replicate comprised of 16 plots $(1 \times 3)$ meters; $50 \mathrm{~cm}$ apart and $150 \mathrm{~cm}$ between irrigated and un-irrigated plots. Four promising bread wheat cultivars i.e. Abu-Ghraib/3, Tamouz/2, IPA/99 and Iraq were assigned to sub-sub plot, two nitrogen rates namely zero and $80 \mathrm{~kg}$ $\mathrm{N} / \mathrm{ha}$ as urea $45 \% \mathrm{~N}$ ) were partitioned in two equal doses, the first dose was added during seed sowing and the second was added at tillers stage, as sub-plot, under rain alone and with (SI) as a main plot ; (SI) was applied at booting and heading stages at a rate of $25 \mathrm{~mm}$ for each stage using perforated bucket to resemble sprinkler irrigation. Telkaef field was sown before fall first rain at 2011-2003, while Mosul field was sown at 11-12-2003 after fall of approximately $75 \mathrm{~mm}$ rain. The seeds were sown in plots as 5 rows, $20 \mathrm{~cm}$ between rows at a depth of 3-4 cm and at a rate of $100 \mathrm{~kg} / \mathrm{ha}$. The recorded characters were plant height, number of effective tillers $/ \mathrm{m}^{2}$ and grain yield $\mathrm{kg} / \mathrm{ha}$. The obtained grains from each treatment were sieved and cleaned manually. Grain nitrogen content was determined by using Micro Kjeldahl method according to (AACC 46-11, 1976) the crude protein percentage was calculated by multiplying the nitrogen percentage by nitrogen factor(5.7). Grain sample for each treatment was prepared and tempered to $15.5 \%$ moisture, thereafter, $100 \mathrm{~g}$ of tempered wheat was ground using (Quadrumat Junior Mill, Germany), and finally sodium dodecyle sulphate sedimentation was calculated according to Axford et al.(1979).

\section{RESULT AND DISCUSSION}

Yield components: Application of nitrogen resulted in insignificant enhancement in number of spikes $/ \mathrm{m}^{2}$, number of grain/spike at both locations (Tables, 1and 2), similar results have been reported by Alkaff and Ba-Momin (1998) and Singh et al.(2003). Supplemental irrigation led to a significant increment in number of grain/spike and insignificant increment in number of spike $/ \mathrm{m}^{2}$ at Telkaef which was planted 22 days before Mosul site. Such results were argued by Al-Ma-mury (1986) Al-Rawi and Al-Hadethi (1988) and Alam et al.( 2003 ). Cultivars showed significant differences in these characters at both 
locations, IPA-99 variety recorded highest number of spike $/ \mathrm{m}^{2}$ at Telkaef site, while Iraq cultivar gave highest value for number of grain/spike at both locations which was significantly different from other cultivars this was related with reduction in number of spike/ $\mathrm{m}^{2}$ which led to minimize the competition on nutrient absorption from soil and those synthesized in plant as well. These results are

general agreement to those of Klar and Hossokawa (1996) and Saleem et al. (2003) . The effect of interaction between irrigation and fertilizer was insignificant for number of spikes $/ \mathrm{m}^{2}$ at Telkaef location, although it caused a remarkable increment with (SI) or $\mathrm{N}$ fertilizer or both which was significant at Mosul location, maximum number of spike $/ \mathrm{m}^{2}$ was recorded for the combination of (SI) with $\mathrm{N}$ fertilizer which was (341.8-380) spikes $/ \mathrm{m}^{2}$ at Telkaef and Mosul respectively, number of grain/spike was increased at both locations with (SI) alone or (SI) with $\mathrm{N}$ fertilizer which reached 51.28 and 42.51 at Telkaef and Mosul, while least number of grain/spike was recorded with no irrigation and fertilizer which was 42.72 and 39.07 for both locations, respectively. The interaction between irrigation with cultivars was significant for most characters at both locations. IPA-99 gave highest number of spike $/ \mathrm{m}^{2}$ with (SI) at Telkaef, while Abu-Ghraib-3 was superior to other cultivars at Mosul site. The Least values were recorded for Iraq cultivar at both locations. This was inversely reflected on number of grain/spike, therefore, Iraq cultivar gave the highest number of grain/spike at both locations, as a result of decrease in number of spike $/ \mathrm{m}^{2}$ and weak competition on nutrient, the reverse was true. The interaction of fertilizer with cultivars behaved similar trend of the interaction of (SI) with cultivars. The second order interaction (cultivars $\times$ nitrogen $\times$ irrigation) showed that number of spikes $/ \mathrm{m}^{2}$, number of grain/spike increased gradually by adding $\mathrm{N}$ fertilizer, supplemental irrigation or both for all cultivars.

Grain yield: Grain yield increased significantly due to nitrogen application at both locations (Table 3), the percentage of such enhancement was $13.02 \%$ and $8.6 \%$ at Telkaef and Mosul site respectively. These results were confirmed by Ottman et al. (2000); Al-Haidary (2003); Boehm et al.(2004); and Hossain et al.(2004).

Table (1) : Effect of Nitrogen level, supplementary irrigation, cultivars and their interaction on number of spike/ $/ \mathrm{m}^{2}$.

\begin{tabular}{|c|c|c|c|c|c|c|c|c|}
\hline \multirow[b]{2}{*}{ Water level } & \multirow[b]{2}{*}{$\begin{array}{c}\text { Nitrogen } \\
\text { level }\end{array}$} & \multicolumn{4}{|c|}{ Wheat cultivar } & \multirow{2}{*}{$\begin{array}{c}\text { Water level } \\
\mathrm{x} \text { nitrogen } \\
\text { level }\end{array}$} & \multirow[b]{2}{*}{$\begin{array}{l}\text { Water level } \\
\text { means }\end{array}$} & \multirow[b]{2}{*}{$\begin{array}{c}\text { Nitrogen } \\
\text { means }\end{array}$} \\
\hline & & $\begin{array}{c}\text { Abu- } \\
\text { Ghraib3 }\end{array}$ & IPA-99 & Tamouz-2 & Iraq & & & \\
\hline \multicolumn{9}{|c|}{ Telkafe } \\
\hline \multirow{2}{*}{ Rain-fed } & Zero N & $312.3 \mathrm{ab}$ & $329.3 \mathrm{ab}$ & 318.0ab & $243.6 \mathrm{c}$ & $301.0 \mathrm{a}$ & & \\
\hline & $80 \mathrm{Kg} \mathrm{N} / \mathrm{ha}$ & $328.0 \mathrm{ab}$ & $351.3 \mathrm{a}$ & $334.6 \mathrm{ab}$ & $259.3 \mathrm{c}$ & $318.3 \mathrm{a}$ & & \\
\hline \multirow{2}{*}{$\begin{array}{c}\text { Rain- } \\
\text { fed+(SI) }\end{array}$} & Zero $\mathrm{N}$ & $337.6 \mathrm{a}$ & $349.3 \mathrm{a}$ & $341.3 \mathrm{a}$ & $264.0 \mathrm{c}$ & $323.0 \mathrm{a}$ & & \\
\hline & $80 \mathrm{Kg} \mathrm{N} / \mathrm{ha}$ & $360.6 \mathrm{a}$ & $360.3 \mathrm{a}$ & $362.0 \mathrm{a}$ & $284.3 \mathrm{bc}$ & $341.8 \mathrm{a}$ & & \\
\hline Rain-fed & & $320.1 \mathrm{a}$ & $340.3 a$ & $326.6 \mathrm{a}$ & $251.5 b$ & & $309.6 a$ & \\
\hline \multirow[t]{3}{*}{$\begin{array}{c}\text { Rain-fed + } \\
\text { (SI) }\end{array}$} & & $349.1 \mathrm{a}$ & $354.8 \mathrm{a}$ & $351.6 \mathrm{a}$ & \multicolumn{2}{|l|}{$274.1 \mathrm{~b}$} & \multirow[t]{3}{*}{$332.4 \mathrm{a}$} & \\
\hline & Zero N & $325.0 \mathrm{a}$ & $339.3 \mathrm{a}$ & $330.0 \mathrm{a}$ & $253.8 b$ & & & $312.0 \mathrm{a}$ \\
\hline & $80 \mathrm{Kg} \mathrm{N} / \mathrm{ha}$ & $344.3 \mathrm{a}$ & $355.8 \mathrm{a}$ & $348.3 a$ & $271.8 \mathrm{~b}$ & & & $330.0 \mathrm{a}$ \\
\hline
\end{tabular}




\begin{tabular}{|c|c|c|c|c|c|c|c|c|}
\hline Wheat cult & ivar means & $334.6 \mathrm{a}$ & $347.5 \mathrm{a}$ & $339.1 \mathrm{a}$ & $262.8 b$ & \multicolumn{2}{|c|}{ Over all mean } & 321.0 \\
\hline \multicolumn{9}{|c|}{ Mosul } \\
\hline \multirow{2}{*}{ Rain-fed } & Zero N & $368.3 \mathrm{~cd}$ & $334.3 \mathrm{de}$ & 331.3de & $255.3 \mathrm{f}$ & \multirow{4}{*}{$\begin{array}{c}322.3 \mathrm{c} \\
343 \mathrm{bc} \\
361.7 \mathrm{ab} \\
380.0 \mathrm{a}\end{array}$} & & \\
\hline & $80 \mathrm{Kg} \mathrm{N} / \mathrm{ha}$ & $393.0 \mathrm{a}-\mathrm{c}$ & $364.3 \mathrm{bd}$ & $353.0 \mathrm{~cd}$ & $264.6 f$ & & & \\
\hline \multirow{2}{*}{$\begin{array}{c}\text { Rain- } \\
\text { fed+(SI) }\end{array}$} & Zero $\mathrm{N}$ & 397.3a-c & $378.3 a-d$ & $392.0 \mathrm{a}-\mathrm{c}$ & $279.3 f$ & & & \\
\hline & $80 \mathrm{Kg} \mathrm{N} / \mathrm{ha}$ & $421.6 \mathrm{a}$ & $399.3 a-c$ & $412.3 \mathrm{ab}$ & $287.0 \mathrm{ef}$ & & & \\
\hline Rain-fed & & $380.6 a b$ & $349.3 b c$ & $342.1 \mathrm{c}$ & $260.0 \mathrm{~d}$ & & $333.0 \mathrm{a}$ & \\
\hline \multirow[t]{3}{*}{$\begin{array}{c}\text { Rain-fed + } \\
(\mathrm{SI})\end{array}$} & & $409.5 a$ & $388.8 \mathrm{a}$ & $402.1 \mathrm{a}$ & $283.1 \mathrm{~d}$ & & $370.9 a$ & \\
\hline & Zero $\mathrm{N}$ & \multirow{2}{*}{$\begin{array}{c}382.8 \mathrm{ab} \\
407.3 \mathrm{a}\end{array}$} & \multirow{2}{*}{$\begin{array}{c}356.3 \mathrm{~b} \\
381.8 \mathrm{ab}\end{array}$} & \multirow{2}{*}{$\begin{array}{c}361.6 \mathrm{~b} \\
382.6 \mathrm{ab}\end{array}$} & \multirow{2}{*}{$\begin{array}{l}267.3 \mathrm{c} \\
275.8 \mathrm{c} \\
\end{array}$} & & & $342.0 \mathrm{a}$ \\
\hline & $80 \mathrm{Kg} \mathrm{N} / \mathrm{ha}$ & & & & & & & $361.9 \mathrm{a}$ \\
\hline \multicolumn{2}{|c|}{ Wheat cultivar means } & $395.0 \mathrm{a}$ & $369.0 \mathrm{~b}$ & $372.1 \mathrm{~b}$ & $271.5 \mathrm{c}$ & \multicolumn{2}{|c|}{ Over all mean } & 351.9 \\
\hline
\end{tabular}

Table (2) : Effect of Nitrogen level, supplementary irrigation, cultivars and their interaction on number of grain/spike

\begin{tabular}{|c|c|c|c|c|c|c|c|c|}
\hline \multirow[b]{2}{*}{ Water level } & \multirow[b]{2}{*}{$\begin{array}{l}\text { Nitrogen } \\
\text { level }\end{array}$} & \multicolumn{4}{|c|}{ Wheat cultivar } & \multirow{2}{*}{$\begin{array}{c}\text { Water level } \\
\mathrm{x} \text { nitrogen } \\
\text { level }\end{array}$} & \multirow[b]{2}{*}{$\begin{array}{c}\text { Water level } \\
\text { means }\end{array}$} & \multirow[b]{2}{*}{$\begin{array}{c}\text { Nitrogen } \\
\text { means }\end{array}$} \\
\hline & & $\begin{array}{c}\text { Abu- } \\
\text { Ghraib3 }\end{array}$ & IPA-99 & Tamouz-2 & Iraq & & & \\
\hline \multicolumn{9}{|c|}{ Telkafe } \\
\hline \multirow{2}{*}{ Rain-fed } & Zero N & $42.09 \mathrm{~d}-\mathrm{f}$ & $44.90 \mathrm{c}-\mathrm{f}$ & $39.8 \mathrm{e}$ & $44.60 \mathrm{c}-\mathrm{f}$ & \multirow{4}{*}{$\begin{array}{c}42.72 \mathrm{~b} \\
44.40 \mathrm{~b} \\
46.09 \mathrm{ab} \\
51.28 \mathrm{a}\end{array}$} & & \\
\hline & $80 \mathrm{Kg} \mathrm{N} / \mathrm{ha}$ & $45.07 \mathrm{c}-\mathrm{f}$ & $45.40 \mathrm{c}-\mathrm{f}$ & 40.19 ef & $46.93 \mathrm{~b}-\mathrm{d}$ & & & \\
\hline \multirow{2}{*}{$\begin{array}{c}\text { Rain- } \\
\text { fed+(SI) }\end{array}$} & \begin{tabular}{|l|} 
Zero $\mathrm{N}$ \\
\end{tabular} & $46.29 \mathrm{~b}-\mathrm{e}$ & $46.39 \mathrm{~b}-\mathrm{e}$ & $44.72 \mathrm{c}-\mathrm{f}$ & $46.96 \mathrm{~b}-\mathrm{d}$ & & & \\
\hline & $80 \mathrm{Kg} \mathrm{N} / \mathrm{ha}$ & $50.97 \mathrm{a}-\mathrm{c}$ & $52.08 \mathrm{ab}$ & $49.00 \mathrm{a}-\mathrm{c}$ & $53.17 \mathrm{a}$ & & & \\
\hline Rain-fed & & \multirow{2}{*}{$\begin{array}{l}43.85 \mathrm{c} \\
48.58 \mathrm{ab}\end{array}$} & $45.15 b c$ & $39.73 d$ & $45.76 b c$ & & $43.56 \mathrm{~b}$ & \\
\hline \multirow[t]{3}{*}{$\begin{array}{c}\text { Rain-fed + } \\
\text { (SI). }\end{array}$} & & & $49.24 \mathrm{ab}$ & $46.86 a-c$ & $50.07 \mathrm{a}$ & & $48.68 \mathrm{a}$ & \\
\hline & Zero $\mathrm{N}$ & \multirow{2}{*}{$\begin{array}{l}44.19 \mathrm{~cd} \\
47.97 \mathrm{a}-\mathrm{c}\end{array}$} & $45.65 \mathrm{~b}-\mathrm{d}$ & \multirow{2}{*}{$\begin{array}{c}42.00 \mathrm{~d} \\
44.60 \mathrm{~b}-\mathrm{d}\end{array}$} & \multirow{2}{*}{$\begin{array}{c}45.78 \mathrm{~b}-\mathrm{d} \\
50.05 \mathrm{a} \\
\end{array}$} & & & $44.40 \mathrm{a}$ \\
\hline & $80 \mathrm{Kg} \mathrm{N} / \mathrm{ha}$ & & $48.74 \mathrm{ab}$ & & & & & $47.84 \mathrm{a}$ \\
\hline \multicolumn{2}{|c|}{ Wheat cultivar means } & $46.08 \mathrm{a}$ & $47.19 \mathrm{a}$ & $43.30 \mathrm{~b}$ & $47.91 \mathrm{a}$ & \multicolumn{2}{|c|}{ Over all mean } & 46.12 \\
\hline \multicolumn{9}{|c|}{ Mosul } \\
\hline \multirow{2}{*}{ Rain-fed } & Zero N & $34.83 \mathrm{~d}$ & $41.13 b c$ & $36.96 \mathrm{~d}$ & 43.36ab & \multirow{4}{*}{$\begin{array}{c}39.07 \mathrm{~b} \\
39.59 \mathrm{~b} \\
40.23 \mathrm{ab} \\
42.51 \mathrm{a}\end{array}$} & & \\
\hline & $80 \mathrm{Kg} \mathrm{N} / \mathrm{ha}$ & $35.06 \mathrm{~d}$ & $41.20 \mathrm{bc}$ & $37.66 \mathrm{~cd}$ & $44.43 \mathrm{ab}$ & & & \\
\hline \multirow{2}{*}{$\begin{array}{c}\text { Rain- } \\
\text { fed+(SI) }\end{array}$} & Zero N & $37.13 \mathrm{~d}$ & $41.26 \mathrm{bc}$ & $37.96 \mathrm{~cd}$ & $44.56 \mathrm{ab}$ & & & \\
\hline & $80 \mathrm{Kg} \mathrm{N} / \mathrm{ha}$ & $37.26 \mathrm{~d}$ & $45.46 \mathrm{a}$ & $41.20 \mathrm{bc}$ & $46.13 \mathrm{a}$ & & & \\
\hline Rain-fed & & $34.95 \mathrm{e}$ & $41.16 \mathrm{bc}$ & $37.31 \mathrm{de}$ & $43.90 \mathrm{a}$ & & $39.33 \mathrm{a}$ & \\
\hline \multirow[t]{3}{*}{$\begin{array}{c}\text { Rain-fed + } \\
\quad(\mathrm{SI})\end{array}$} & & $37.20 \mathrm{de}$ & $43.36 \mathrm{ab}$ & $39.58 \mathrm{~cd}$ & $45.35 \mathrm{a}$ & & $41.37 \mathrm{a}$ & \\
\hline & Zero N & \multirow{2}{*}{$\begin{array}{l}35.98 \mathrm{e} \\
36.16 \mathrm{e}\end{array}$} & $41.20 \mathrm{bc}$ & $37.46 \mathrm{de}$ & $43.96 \mathrm{a}$ & & & $39.65 \mathrm{a}$ \\
\hline & $80 \mathrm{Kg} \mathrm{N} / \mathrm{ha}$ & & $43.33 \mathrm{ab}$ & $39.43 \mathrm{~cd}$ & $45.20 \mathrm{a}$ & & & $41.05 \mathrm{a}$ \\
\hline Wheat cult & ivar means & $36.07 \mathrm{~d}$ & $42.26 \mathrm{~b}$ & $38.49 \mathrm{c}$ & $44.62 \mathrm{a}$ & Over: & all mean & 40.35 \\
\hline
\end{tabular}

Supplemental irrigation enhanced grain yield at both locations, the enhancement was 23.7 and $18.1 \%$ for Telkaf and Mosul respectively. These results are in general agreement with those of Engel et al. (2001); and Alam et al. (2003). It is obvious that

the effect of (SI) was approximately double of that caused by nitrogen application. It is well known that the moisture is the major limiting factor determining grain yield potential under arid and semi-arid regions .Cultivars were also significantly different in grain yield, IPA-99 gave highest grain yield at both location which were 4623, $4250 \mathrm{~kg} / \mathrm{ha}$ at Telkaef and Mosul, respectively, while minimum yield was for Abu-Ghraib/3 and Iraq at both location. Differences between cultivars have been also reported by Klar and Hossokawa (1996) AlSamaray (2002) Boehm et al. (2004) Beuerlein et al. (2004). Such differences are mainly due to their genetic potential, production capacity, and efficiency in speed of nitrogen absorption. Nitrogen fertilizer and irrigation interaction 
significantly influenced grain yield at both locations they caused a linear increment with nitrogen or (SI) or both in comparison to control. Garabet et al. (1998b); and Oweis et al. (1998) obtained higher yield with nitrogen fertilizer and supplementary irrigation similarly, moreover Oweis et al. (2000) reported that such application should be combined with proper sowing date, which is obvious in the present study particularly at Telkaef location, since it was sown earlier than Mosul location. Cultivars by irrigation interaction was significantly affected grain yield, IPA-99 and Tamouz-2 gave higher yield with (SI) at both location, also all cultivars respond positively with nitrogen application at both location, similar results have been reported by Singh et al. (2003). The second order interaction caused a gradual increase of yield in all cultivars with nitrogen or supplemental irrigation or both, and the highest value was for IPA-99 with nitrogen and irrigation for both locations. The supplemental irrigation improved the efficiency of nitrogen utilization, but the response depends on the cultivar absorbing ability, so the combination of the three factors is very important to achieve maximum benefits of increasing grain yield.

Table (3) : Effect of Nitrogen level, supplementary irrigation, cultivars and their interaction on grain yield $\mathrm{kg} / \mathrm{ha}$.

\begin{tabular}{|c|c|c|c|c|c|c|c|c|}
\hline \multirow[b]{2}{*}{ Water level } & \multirow{2}{*}{$\begin{array}{l}\text { Nitrogen } \\
\text { level }\end{array}$} & \multicolumn{4}{|c|}{ Wheat cultivar } & \multirow{2}{*}{$\begin{array}{c}\text { Water level } \\
\mathrm{x} \text { nitrogen } \\
\text { level }\end{array}$} & \multirow{2}{*}{$\begin{array}{c}\text { Water level } \\
\text { means }\end{array}$} & \multirow{2}{*}{$\begin{array}{c}\text { Nitrogen } \\
\text { means }\end{array}$} \\
\hline & & $\begin{array}{c}\text { Abu- } \\
\text { Ghraib3 }\end{array}$ & IPA-99 & Tamouz-2 & Iraq & & & \\
\hline \multicolumn{9}{|c|}{ Telkafe } \\
\hline \multirow{2}{*}{ Rain-fed } & Zero N & 3549 gh & 3948 ef & $3761 \mathrm{gh}$ & $3497 \mathrm{~h}$ & \multirow{4}{*}{$\begin{array}{l}3688 \mathrm{~d} \\
4150 \mathrm{c} \\
4543 \mathrm{~b} \\
5155 \mathrm{a}\end{array}$} & & \\
\hline & $80 \mathrm{Kg} \mathrm{N} / \mathrm{ha}$ & 3998 ef & $4489 \mathrm{~cd}$ & $4205 \mathrm{de}$ & 3906 e-g & & & \\
\hline \multirow{2}{*}{$\begin{array}{c}\text { Rain- } \\
\text { fed+(SI) }\end{array}$} & Zero N & $4484 \mathrm{~cd}$ & $4725 \mathrm{bc}$ & $4680 \mathrm{bc}$ & $4285 \mathrm{de}$ & & & \\
\hline & $80 \mathrm{Kg} \mathrm{N} / \mathrm{ha}$ & $5068 \mathrm{ab}$ & $5331 \mathrm{a}$ & $5223 \mathrm{a}$ & $4998 \mathrm{ab}$ & & & \\
\hline Rain-fed & & $3773 \mathrm{de}$ & $4218 \mathrm{c}$ & $3983 \mathrm{~cd}$ & $3701 \mathrm{e}$ & & $3919 \mathrm{~b}$ & \\
\hline \multirow[t]{3}{*}{\begin{tabular}{|c|}
$\begin{array}{c}\text { Rain-fed + } \\
\text { (SI). }\end{array}$ \\
\end{tabular}} & & $4776 \mathrm{ab}$ & $5028 \mathrm{a}$ & $4951 \mathrm{a}$ & \multicolumn{2}{|l|}{$4641 \mathrm{~b}$} & \multirow[t]{3}{*}{$4849 a$} & \\
\hline & Zero $\mathrm{N}$ & 4016 de & $4336 \mathrm{~cd}$ & $4220 \mathrm{de}$ & $3891 \mathrm{e}$ & & & $4116 \mathrm{~b}$ \\
\hline & $80 \mathrm{Kg} \mathrm{N} / \mathrm{ha}$ & $4533 \mathrm{bc}$ & $4910 \mathrm{a}$ & $4714 \mathrm{ab}$ & $4452 \mathrm{bd}$ & & & $4652 \mathrm{a}$ \\
\hline \multicolumn{2}{|c|}{ Wheat cultivar means } & $4275 \mathrm{~b}$ & $4623 \mathrm{a}$ & $4467 \mathrm{a}$ & $4171 \mathrm{~b}$ & \multicolumn{2}{|c|}{ Over all mean } & 4384 \\
\hline \multicolumn{9}{|c|}{ Mosul } \\
\hline \multirow{2}{*}{ Rain-fed } & Zero $\mathrm{N}$ & $3503 \mathrm{~g}$ & 3910 e-g & $3814 \mathrm{fg}$ & $3570 \mathrm{~g}$ & \multirow{4}{*}{$\begin{array}{l}3699 \mathrm{~d} \\
4063 \mathrm{c} \\
4417 \mathrm{~b} \\
4750 \mathrm{a}\end{array}$} & & \\
\hline & $80 \mathrm{Kg} \mathrm{N} / \mathrm{ha}$ & $3834 \mathrm{e}-\mathrm{g}$ & $4327 \mathrm{bc}$ & $4232 \mathrm{~cd}$ & $3859 \mathrm{fg}$ & & & \\
\hline \multirow{2}{*}{$\begin{array}{c}\text { Rain- } \\
\text { fed+(SI) }\end{array}$} & Zero N & $4166 \mathrm{de}$ & $4715 b$ & $4636 \mathrm{bc}$ & $4154 \mathrm{~d}-\mathrm{f}$ & & & \\
\hline & $80 \mathrm{Kg} \mathrm{N} / \mathrm{ha}$ & $4367 \mathrm{~b}-\mathrm{d}$ & $5130 \mathrm{a}$ & $5125 \mathrm{a}$ & 4380b-d & & & \\
\hline Rain-fed & & $3669 \mathrm{c}$ & $4118 b$ & $4023 \mathrm{~b}$ & $3714 \mathrm{c}$ & \multicolumn{2}{|r|}{$3881 \mathrm{~b}$} & \\
\hline \multirow[t]{3}{*}{$\begin{array}{l}\text { Rain-fed + } \\
\text { (SI) }\end{array}$} & & $4266 \mathrm{~b}$ & $4922 \mathrm{a}$ & $4880 \mathrm{a}$ & $4267 \mathrm{~b}$ & & $4584 \mathrm{a}$ & \\
\hline & Zero $\mathrm{N}$ & \multirow{2}{*}{$\begin{array}{c}3834 \mathrm{c} \\
4101 \mathrm{bc}\end{array}$} & $4312 b$ & $4225 \mathrm{~b}$ & \multirow{2}{*}{$\begin{array}{c}3862 \mathrm{c} \\
4120 \mathrm{bc}\end{array}$} & & & $4058 \mathrm{~b}$ \\
\hline & $80 \mathrm{Kg} \mathrm{N} / \mathrm{ha}$ & & $4728 \mathrm{a}$ & $4678 \mathrm{a}$ & & & & $4407 \mathrm{a}$ \\
\hline \multicolumn{2}{|c|}{ Wheat cultivar means } & $3967 \mathrm{~b}$ & $4250 \mathrm{a}$ & $4451 \mathrm{a}$ & $3991 b$ & \multicolumn{2}{|c|}{ Over all mean } & 4232 \\
\hline
\end{tabular}

Protein content $\%$ and sedimentation value: Application of nitrogen increased significantly protein percentage at both locations, such results are in general agreement to those of Abbasi et al. (1991); Teama et al. (1993) and Ottman et al. (2000). Sedimentation value was similarly increased referring to association of sedimentation value with protein \%. Irrigation has no influence on protein or sedimentation value at both locations (Tables 4 and 5). Wheat cultivars showed a significant difference in protein $\%$, Iraq cultivar recorded highest value which 
was 14.03 and $14.31 \%$ at Telkaef and Mosul, respectively; the lowest was for IPA-99 which was 13.11 and $13.25 \%$ at both locations, meanwhile Abu-Ghraib/3 and Iraq varieties recorded highest values of sedimentation at both locations which due to their higher protein content. Fertilizer and irrigation also affected these parameters significantly highest protein $\%$ and sedimentation value was for the treatment under rainfall with $\mathrm{N}$ fertilizer at both locations. Irrigation with cultivars interaction have a significant effect on protein $\%$ and sedimentation value, Iraq cultivar under (SI) gave highest protein \% and sedimentation value. These results are supported by Al-Ani (1993)who reported that availability of adequate amount of moisture in soil caused increment in nitrogen uptake due to superior growth and roots penetration which increase total root depletion zone and led to absorb greater nitrogen by plant. Cultivars respond differently to $\mathrm{N}$ fertilizer at both locations in respect to protein \%, Iraq cultivar showed highest protein \% with $\mathrm{N}$ fertilizer which was 14.26 and 14.5 at Telkaef and Mosul respectively,

Table (4) : Effect of Nitrogen level, supplementary irrigation, cultivars and their interaction on Seed protein \% .

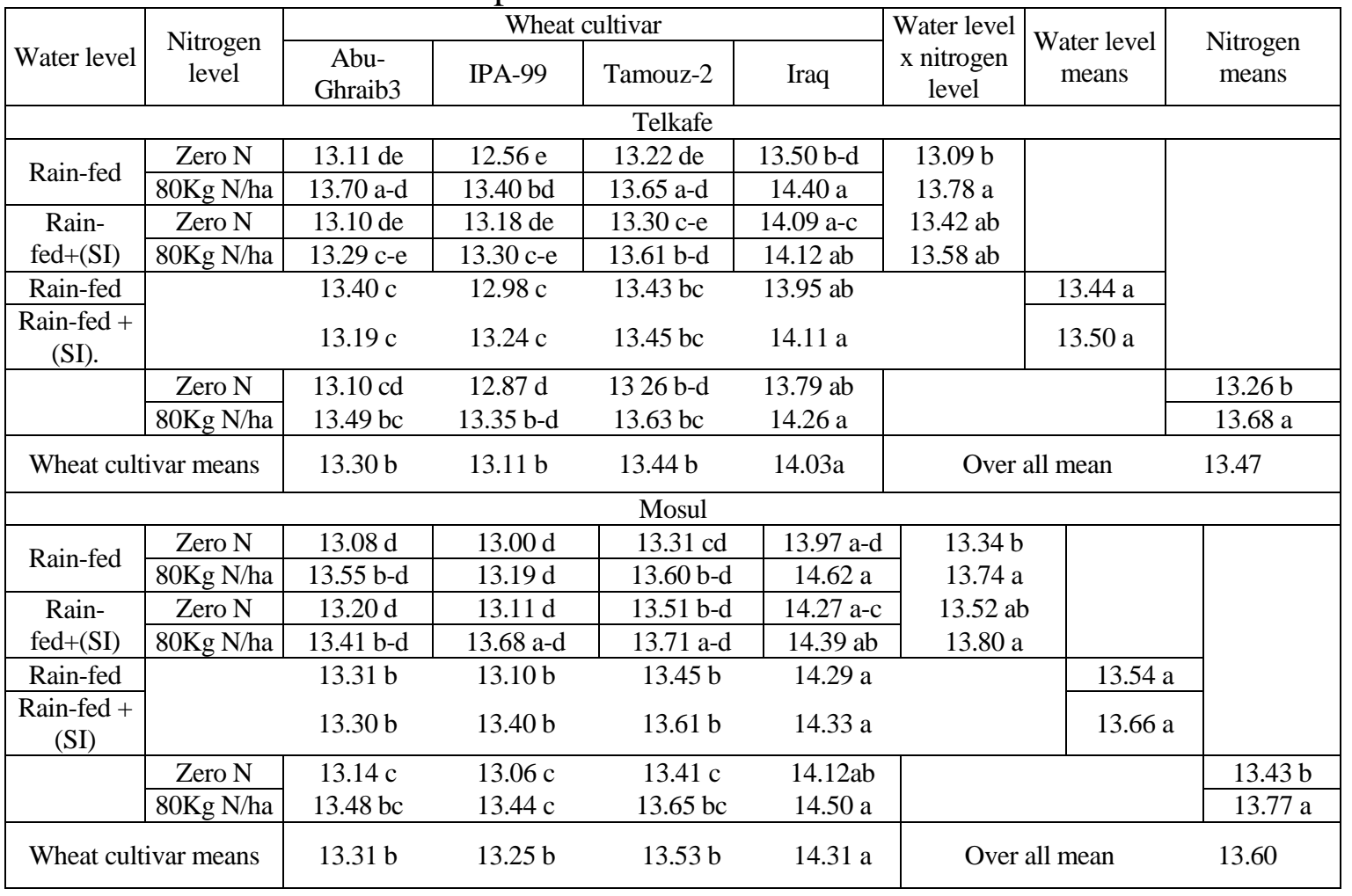

Table (5): Effect of Nitrogen level, supplementary irrigation, cultivars and their interaction on Sedimentation value $/ \mathrm{cm}^{3}$

\begin{tabular}{|c|c|c|c|c|c|c|c|c|}
\hline \multirow[b]{2}{*}{ Water level } & \multirow[b]{2}{*}{$\begin{array}{l}\text { Nitrogen } \\
\text { level }\end{array}$} & \multicolumn{4}{|c|}{ Wheat cultivar } & \multirow{2}{*}{$\begin{array}{l}\text { Water level } \\
\text { x nitrogen } \\
\text { level }\end{array}$} & \multirow[b]{2}{*}{$\begin{array}{c}\text { Water level } \\
\text { means }\end{array}$} & \multirow{2}{*}{$\begin{array}{c}\text { Nitroger } \\
\text { means }\end{array}$} \\
\hline & & $\begin{array}{c}\text { Abu- } \\
\text { Ghraib3 }\end{array}$ & IPA-99 & Tamouz-2 & Iraq & & & \\
\hline \multicolumn{9}{|c|}{ Telkafe } \\
\hline \multirow{2}{*}{ Rain-fed } & Zero $\mathrm{N}$ & $71.1 \mathrm{c}-\mathrm{e}$ & $68.60 \mathrm{~h}$ & $70.56 \mathrm{~d}-\mathrm{f}$ & $71.46 \mathrm{~b}-\mathrm{e}$ & $70.43 b$ & & \\
\hline & $80 \mathrm{Kg} \mathrm{N} / \mathrm{ha}$ & $73.60 \mathrm{a}$ & $68.76 \mathrm{~h}$ & $71.80 \mathrm{~b}-\mathrm{d}$ & $73.23 \mathrm{a}$ & $71.95 \mathrm{a}$ & & \\
\hline \multirow{2}{*}{$\begin{array}{c}\text { Rain- } \\
\text { fed+(SI) }\end{array}$} & Zero $\mathrm{N}$ & $72.00 \mathrm{~b}-\mathrm{d}$ & $69.03 \mathrm{gh}$ & $69.53 \mathrm{f}-\mathrm{h}$ & $72.03 \mathrm{bc}$ & $70.65 \mathrm{~b}$ & & \\
\hline & $80 \mathrm{Kg} \mathrm{N} / \mathrm{ha}$ & $73.43 \mathrm{a}$ & $70.23 \mathrm{e}-\mathrm{g}$ & $70.93 \mathrm{c}-\mathrm{e}$ & $72.83 \mathrm{ab}$ & $71.85 \mathrm{a}$ & & \\
\hline Rain-fed & & $72.35 \mathrm{a}$ & $68.68 \mathrm{~d}$ & $71.18 \mathrm{~b}$ & $72.55 \mathrm{a}$ & & $71.19 \mathrm{a}$ & \\
\hline
\end{tabular}




\begin{tabular}{|c|c|c|c|c|c|c|c|c|}
\hline $\begin{array}{c}\text { Rain-fed + } \\
\text { (SI). }\end{array}$ & & $72.71 \mathrm{a}$ & $69.63 \mathrm{c}$ & $70.23 \mathrm{c}$ & \multicolumn{2}{|l|}{$72.43 \mathrm{a}$} & $71.25 \mathrm{a}$ & \multirow[b]{2}{*}{$70.54 \mathrm{~b}$} \\
\hline & Zero $\mathrm{N}$ & $71.55 \mathrm{~b}$ & $68.81 \mathrm{~d}$ & $70.05 \mathrm{c}$ & $71.78 \mathrm{~b}$ & & & \\
\hline & $80 \mathrm{Kg} \mathrm{N} / \mathrm{ha}$ & $73.51 \mathrm{a}$ & $69.50 \mathrm{~cd}$ & $71.36 \mathrm{~b}$ & $73.23 \mathrm{a}$ & & & $71.90 \mathrm{a}$ \\
\hline Wheat cult & ivar means & $72.53 \mathrm{a}$ & $69.15 \mathrm{c}$ & $70.70 \mathrm{~b}$ & $72.50 \mathrm{a}$ & Over al & mean & 71.22 \\
\hline & & & & Mosul & & & & \\
\hline & Zero N & $71.60 \mathrm{a}-\mathrm{d}$ & $68.76 \mathrm{e}$ & $69.83 \mathrm{c}-\mathrm{e}$ & $71.13 a-f$ & $70.33 \mathrm{c}$ & & \\
\hline Rain-fed & $80 \mathrm{Kg} \mathrm{N} / \mathrm{ha}$ & $72.86 \mathrm{ab}$ & $69.06 \mathrm{e}$ & 70.93 b-e & $73.46 \mathrm{a}$ & $71.58 \mathrm{a}$ & & \\
\hline Rain- & Zero N & 72.16 a-c & $69.76 \mathrm{de}$ & $70.06 \mathrm{c}-\mathrm{e}$ & $72.23 a-c$ & $71.05 \mathrm{~b}$ & & \\
\hline fed $+(\mathrm{SI})$ & $80 \mathrm{Kg} \mathrm{N} / \mathrm{ha}$ & $72.76 \mathrm{ab}$ & $70.00 \mathrm{c}-\mathrm{e}$ & $70.03 \mathrm{c}-\mathrm{e}$ & $72.73 \mathrm{ab}$ & $71.38 \mathrm{ab}$ & & \\
\hline Rain-fed & & $72.23 \mathrm{a}$ & $68.91 \mathrm{~b}$ & $70.38 \mathrm{~b}$ & $72.30 \mathrm{a}$ & & $70.95 \mathrm{a}$ & \\
\hline $\begin{array}{l}\text { Rain-fed + } \\
\quad(\mathrm{SI})\end{array}$ & & $72.46 \mathrm{a}$ & $69.86 \mathrm{~b}$ & $70.05 \mathrm{~b}$ & $72.48 \mathrm{a}$ & & $71.21 \mathrm{a}$ & \\
\hline & Zero $\mathrm{N}$ & $71.88 \mathrm{ab}$ & $69.25 \mathrm{c}$ & $69.95 \mathrm{c}$ & $71.68 \mathrm{ab}$ & & & $70.69 \mathrm{~b}$ \\
\hline & $80 \mathrm{Kg} \mathrm{N} / \mathrm{ha}$ & $72.81 \mathrm{a}$ & $6953 \mathrm{c}$ & $70.48 \mathrm{bc}$ & $73.10 \mathrm{a}$ & & & $71.48 \mathrm{a}$ \\
\hline Wheat cult & ivar means & $72.35 \mathrm{a}$ & $69.39 \mathrm{~b}$ & $70.21 \mathrm{~b}$ & $72.39 \mathrm{a}$ & Over & 11 mean & 71.08 \\
\hline
\end{tabular}

meanwhile IPA-99 gave least protein\% without nitrogen which was 12.87 and 13.06 at both locations. Abu-Ghraib/3 and Iraq cultivar with $\mathrm{N}$ fertilizer recorded highest sedimentation value at both locations. The second order interaction showed that Iraq cultivar under rainfall with $\mathrm{N}$ fertilizer alone gave highest protein \% at both locations which were 14.40 and 14.62\% for Telkaef and Mosul, respectively; while IPA-99 recorded the least value with control which were 12.56 and $13.0 \%$ for the same locations, the highest sedimentation value was for Abu-Ghraib/3 and Iraq with $\mathrm{N}$ fertilizer and without (SI) which were 73.60 and $73.23 \mathrm{ml}$ at Telkaef and 72.86 and $73.46 \mathrm{ml}$ at Mosul, and this was accompanied with protein $\%$ content.

$$
\begin{aligned}
& \text { تأثير التسميد النيتروجين والري التكميلي في الحاصل والصفات النوعية لبعض الأصناف المحلية من } \\
& \text { حنظة الخبز الناعمة والصفات }
\end{aligned}
$$

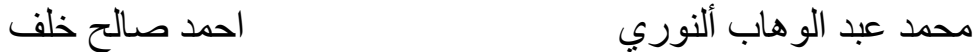

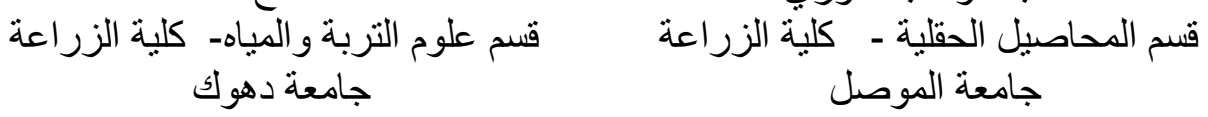

\section{الخلاصة}

تعتمد إنتاجية الحنطة في المناطق الايمية ومنها المنطقة الثمالية في العراق بشكل كبير على كمية

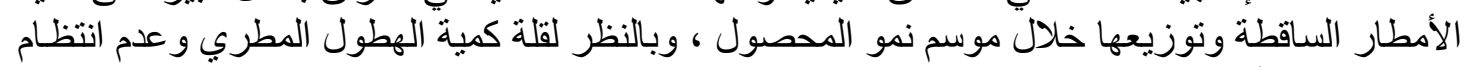

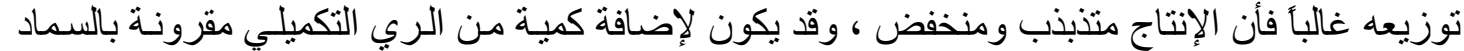

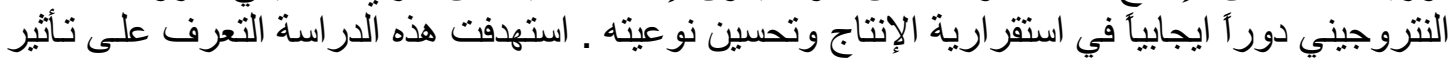



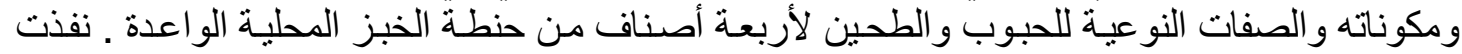

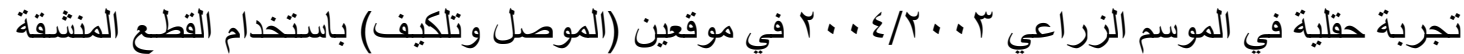

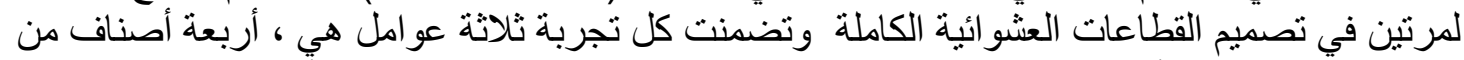

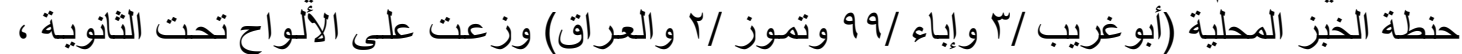

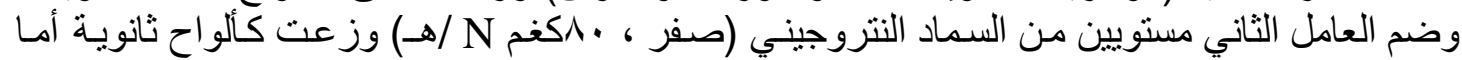

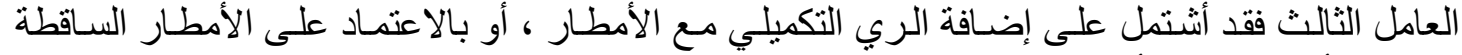

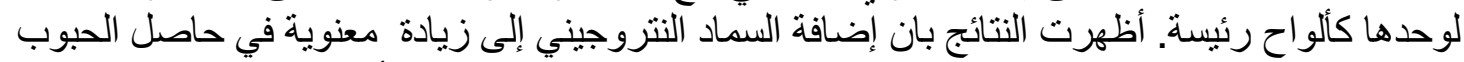






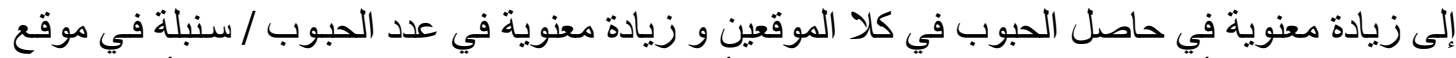

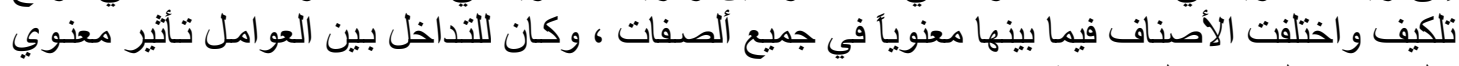
على جميع الصفات المدروسة مانسان

\section{REFERENCES}

AACC (1976). Approved methods of the American Association of Cereal Chemists. St. Paul, Minnesota, USA .

Abbasi, Z. ; M. Kh. Aldermosh ; A. Matar and A. Mekhail (1991). Effect of nitrogen fertilization and supplementary irrigation on the productivity, and protein content of five local and improved wheat cultivars. Research J. of Aleppo Univ. 16:57-74.

Adary , A.; A. Hachum; T. Oweis; and M. Pala (2002). Wheat productivity under supplemental irrigation in Northern Iraq. On-farm water husbandry research report series, No.2. ICARDA Aleppo, Syria.

Alam, M.Z. ;M.S. Rahman; M.E. Haque; M.S. Hossain; M.A.K. Azed; and M.R.H Khan (2003). Response of irrigation frequencies and different doses of N. fertilization on the growth and yield of wheat. Pakistan J. of Biological Sci.6(8): 732-734.

Al-Ani,M.A.M.(1993).The role of biotechnology in growth and production of wheat and soybean ,utilizing VA-Mycorrhiza fungi. A dissertation submitted to the college of Agric. And forestry-Mosul University

Al-Haidary, H. (2003). Effect of time of nitrogen application and seed rate on growth, yield and quality of bread wheat. Ph.D. Thesis. College of Agric. University of Baghdad .

Al-Kaff, H.A. ; and A.M. BA-Momin (1998). Effect of nitrogen and phosphorus application on growth, yield and N.P. content of wheat (cv. Kalyansona) grown under delta Wadi Tuban condition. Yemens Jour. of Agri. Research 11:75-88.

Al-Ma'mury, M.J.(1986). The influence of irrigation methods and soil water regimes on yield and quality of wheat and barley crops. Ms.C. Thesis, Collage of Agric., University of Baghdad .

Al-Rawi,A.A.; and A.A.Al-Hadethi(1988). Effect of irrigation and nitrogen fertilization on the growth and yield of wheat (Triticum aestivum L.V. Mexipak). Mesopotamia J. of Agric. 19(2):56-62.

Al-Samaray, I.K.(2002). Application of complete improvements to raise wheat productivity . Iraqi Agric. J. 1:35-61.

Axford, D.W.E;E.E. Mcdermott; and D.G Redman (1979). Note on the sodium dodecyle sulphate test of bread making quality: comparison with Pelshenke and Zeleny tests . Cereal Chem. 56(6): 582-584.

Beuerlein,J.;P.Lipps, and R.Minyo(2004). 2004 Ohio wheat performance test. OSU, Horticulture and Crop Sci. series 228.

Boehm, D. J; W. A. Berzonsky; and M. Bhattaharya (2004). Influence of nitrogen fertilization treatment on spring wheat (Triticum aestivum L.). Flour characteristics and effect on fresh and frozen dough quality. Cereal Chem. 81(1):51-54. 
Engel, R.(1993). Winter wheat response to available nitrogen and water. Montana State University. MSU. Fertilizer Facts, Feb. 1993, No.4.

Engel, R.; D. Lang; and G.Carlson (2001). Nitrogen requirements and yield potential of spring wheat as affected by water. Montana State University. MSU. Fertilizer fact . Jan.2001 No.25 .

Garabet, S. ; M. Wood ; and J. Rayan (1998a). Nitrogen and water effects on wheat yield in a mediterranean - type climate. 1:Growth, water use and nitrogen accumulation .Field Crops Res. 57:309-310 .

Garabet, S. ; J. Rayan ; and M. Wood (1998b). Nitrogen and water effects on wheat yield in a mediterranean type climate. II: Fertilizer-use efficiency with labeled nitrogen . Field Crops Res. 58:213-221.

Hossain, M.t; G.Meisner; J.M. Duxbury; J.G. Lauren; M.M. Rahman; M.M. Meer; and M.H. Rashid(2004). Use of raised beds for increasing wheat production in rice-wheat cropping systems . $4^{\text {th }}$ international Crop Science Congress.(4ICSC)

Klar,A.E. and T. Hossokawa (1996). Wheat cultivars response to irrigation and sowing dates . Scientia Agricola vol 53N.2-3 Piracicaba .

Moursi, M. A; N. A. Nour El-din; O. H. El-Bagoury; and A. A. El.Sayed (1983). Water requirement of wheat II. Effect of drought conditions and different stages of plant age on growth, yield and grain quality . proc. 15t. conf, Agron. Cairo. Egypt, 1(b): 303-315.

Nosaif, A.I.(2002). Importance of supplemental irrigation in dry land farming . Iraqi

Agric. J. Special No. p.62.

Ottman, M.J.;A.D. Thmas ; and Edward (2000). Durum grain quality as affected by nitrogen fertilization near anthesis and irrigation during grain fill. Agron. J. 92:1035-1041.

Oweis, T.,M.Pala;and J. Ryan (1998). Water use efficiency of rainfall and irrigation bread wheat in a Mediterranean environment . Agron. J. 92(2):231-238.

Oweis, T.Heping; and M.Pala(2000). Water use efficiency of rainfall and irrigated bread wheat in a Mediterranean environments. Agron. J. 22(2):231-238.

Saleem, M. (2003). Reponse of durum and bread wheat genotypes to drought stress: Biomass and yield components. Asian J. of Plant Sci. 2(3):290-293.

Singh, S.S.; G. P. Singh ; J.B. Sharma ; N. Chand ; D.N. Sharma ; J.B. Singh ; P.C. Pandy ; M.R.S. Kaim ; T. Mohapatra ; K.P. Singh (2003). Development of new plant type wheat with increased yield potential: Methodology and response to various levels of fertility. Indian Agr.Res. INC. Division of Genetics p:81-86.

Statistic Annual information issue (2003). Ministry of planning and development corporation . Republic of Iraq .

Teama, E.A., R.A. Dawood; and K.A. Kherialla (1993). Quality response of some spring wheat cultivars to different nitrogen fertilization rates. Ass. J. of Agric. Sci. 24(3):137-161 . 